\title{
Coarctação da Aorta. Anomalia Congênita com Novas Perspectivas de Tratamento
}

\author{
Marco Aurélio Santos, Vitor Manuel Pereira Azevedo
}

Rio de Janeiro, RJ

Coarctação, do latim coartatio, quando se refere ao arco aórtico, normalmente indica uma constrição no istmo aórtico entre a origem da artéria subclávia esquerda e o ductus arteriosus. Evans ${ }^{1}$ descreveu sua ocorrência em uma de cada 1.000 autópsias, sendo que Abbot ${ }^{2}$ relatou incidência de 178 em sua série de 1.000 casos de cardiopatias congênitas.

Esta malformação foi, primariamente, descrita em 1760 por Morgani, e a partir de então, muitos estudos foram realizados. Apesar de se apresentar pura e simplesmente como uma lesão vascular, sua patogênese e seu tratamento ainda permanecem controversos. Entre os fatores responsáveis por essas controvérsias, a dificuldade nas relações anatômicas e a influência do fechamento doductus arteriosus talvez sejam as principais.

Existem duas lesões anatômicas que causam limitação ao fluxo aórtico no seu arco. São a coarctação ístmica e a hipoplasia tubular. Freqüentemente, estas duas doenças são rotuladas como coarctação da aorta, porém, muitas vezes faz-se necessário uma avaliação mais precisa ${ }^{3}$.

A coarctação ístmica é representada por lesão obstrutiva da parede do arco aórtico na porção distal do istmo, na junção do ductus arteriosus com a aorta descendente. Comumente, a parede posterior da aorta distal à constrição é caracterizada por uma grande placa da íntima denominada por Edwards e cols. ${ }^{4}$ de "lesão de jato", sendo o local de choque do fluxo sangüíneo que passa através do reduzido orifício. A aorta assume configuração de ampulheta com dilatação proximal de diâmetro próximo ao diâmetro distal à zona de constrição ${ }^{5}$. Já a hipoplasia tubular representa a persistência da configuração fetal do istmo aórtico ${ }^{6}$, apresentando-se como uma longa e difusa zona de coarctação

Instituto Nacional de Cardiologia Laranjeiras

Correspondência: Marco Aurélio Santos - Rua Bulhões de Carvalho, 245/301 22081-000 - Rio de Janeiro, RJ - E-mail: masantos@cardiol.br

Recebido para publicação em 25/10/01

Aceito em $6 / 5 / 02$ no arco aórtico. A hipoplasia tubular pode ou não estar acompanhada de coarctação ístmica.

\section{Classificação}

Uma das primeiras tentativas de classificar a coarctação da aorta foi feita por Bonnet ${ }^{7} \mathrm{em} 1903$ e tornou-se referência. Ele dividiu a coarctação da aorta em dois tipos infantil e adulto, sendo o infantil proximal e o adulto distal ao ductus arteriosus. O tipo infantil está associado à persistência do ductus arteriosus, apresenta estreitamento longo e difuso do istmo aórtico e os pacientes têm mortalidade precoce. Contrariamente, a do tipo adulto está associada à constrição "abrupta", o ductus arteriosus encontra-se fechado, existe grande rede de circulação colateral e os pacientes têm maior sobrevida. Esta classificação, entretanto, está sujeita a crítica, já que a idade de aparecimento dos sintomas nem sempre corresponde aos achados anatômicos. Além disso, não engloba casos de coarctação da aorta em locais infreqüentes. Em 1933, Evans ${ }^{1}$ propôs classificação utilizando as alterações anatômicas bem como a disposição de defeitos associados. Posteriormente, em 1947, Bramwell ${ }^{8}$ simplificou esta classificação em três grupos. Nesse mesmo ano, Reifenstein e cols. ${ }^{9}$ idealizaram classificação baseada no tamanho do diâmetro da luz da aorta ao nível da coarctação - moderada, quando o diâmetro era $\geq 5 \mathrm{~mm}$ e extrema, quando $\leq 5 \mathrm{~mm}$. Entretanto, foi a classificação de Johnson e cols. ${ }^{10}$ que forneceu mais subsídios clínicos e cirúrgicos. Os autores consideraram a persistência do ductus arteriosus, sua relação com o local da coarctação da aorta e a presença ou ausência de circulação colateral, como elementos fundamentais para o manuseio desses pacientes. A essa classificação básica de Johnson e cols., Clagett e cols. ${ }^{11}$ adicionaram variantes clínicas de hipertensão no braço direito com pulsos fracos no braço esquerdo ou hipertensão no braço esquerdo com pulsos fracos no braço direito.

Nestes últimos anos, o termo coarctação da aorta tem sido aplicado mais genericamente como uma constrição, ocupando uma área na junção entre o arco aórtico e a aorta descendente, ou proximal ou distal aoductus arteriosus. $\mathrm{O}$ 
termo justa ductal foi introduzido por Rudolph e cols. ${ }^{12}$ para descrever a constrição da aorta, oposta ao local de origem do ductus arteriosus ou do ligamento arterioso.

Teoria Skodaica-Em 1855 Skoda ${ }^{13}$ sugeriu que a obstrução aórtica era devido à obliteração que teria ocorrido na vida fetal ou uma extensão do tecido ductal na aorta.

Teoria de fluxo - a observação de que a coarctação da aorta, freqüentemente, está associada a malformações cardíacas com baixo fluxo aórtico levou os investigadores a admitir que o baixo fluxo aórtico poderia ser responsável pela origem da coarctação da aorta. Hutchins ${ }^{14}$ propôs que o fluxo pulmonar relativamente maior que o fluxo aórtico predispõe ao desenvolvimento da coarctação da aorta. Duas hipóteses da teoria de fluxo foram propostas por Rudolph e cols. ${ }^{12}: 1^{\mathrm{a}}$, uma discreta reorientação do ângulo que o $d u c$ tus arteriosus faz com a aorta descendente, alteraria a direção da corrente de fluxo ductal contra a parede posterior da aorta e $2^{\mathrm{a}}$, uma vez que as dimensões do sistema aórtico eram determinadas pelo volume de fluxo através dele na fase final da vida fetal, o estreitamento ístmico aórtico normal tornar-se-ia mais acentuado com a obliteração do ductus arteriosus após o nascimento, produzindo uma obstrução ao fluxo, somente após seu fechamento. Shinebourne e Elseed ${ }^{15}$ defendem esta teoria, afirmando que a coarctação da aorta não ocorre em situações em que o fluxo no tronco da artéria pulmonar encontra-se reduzido durante a vida fetal.

Portanto, a coarctação da aorta e, com certa extensão, a hipoplasia tubular, é provavelmente o resultado da junção defeituosa entre o $4^{\circ}$ e o $6^{\circ}$ arco embrionário. A presença de tecido ductal no istmo, provavelmente, predispõe à hipoplasia tubular, especialmente, quando acompanhada por fluxo aórtico baixo.

\section{Tratamento}

Há seis décadas que o tratamento da coarctação da aorta ainda vem apresentando controvérsias, não estando definitivamente bem estabelecido. Desde os relatos iniciais sobre sua correção cirúrgica em $1945^{16,17}$ a literatura vem cada vez mais identificando questões ainda não resolvidas. Uma delas é por que a mortalidade e a morbidade ainda não alcançaram níveis de excelência, principalmente, levando-se em consideração que a coarctação da aorta, teoricamente, é uma malformação de anatomia e fisiologia simples.

Um tratamento corretivo, supostamente, deve proporcionar a manutenção e o funcionamento normal da malformação tratada, oferecer expectativa de vida normal para o paciente e, por fim, posteriores tratamentos clínicos ou cirúrgicos tornar-se-iam desnecessários.

Clarkson e cols. ${ }^{18}$, em seguimento de 10 a 28 anos após cirurgia, demonstraram que somente $20 \%$ dos pacientes operados de coarctação da aorta após um ano de vida, estavam sem qualquer enfermidade cardiovascular residual ou recorrente e que o aparecimento de aneurisma da aorta no local da aortoplastia se desenvolveu até 20 anos após o procedimento.

\section{Tratamento cirúrgico}

Os problemas que envolvem o tratamento da coarctação da aorta estão centrados em três pontos: idade na realização do procedimento, tipo de técnica cirúrgica empregada e anomalias associadas.

Van Heurn e cols. ${ }^{19}$ reviram a experiência de 151 lactentes com idade inferior a três meses, operados entre 1985 e 1990. Nesse grupo, identificaram 60\% de hipoplasia ístmica. Em 77 pacientes, a técnica cirúrgica foi a anastomose término-terminal. Em seguimento de quatro anos, $77 \%$ dos pacientes nos quais foi empregada a técnica término-terminal e, em $51 \%$, a telha de subclávia esquerda, estavam livres de coarctação da aorta. $\mathrm{O}$ aparecimento de recoarctação esteve associada com baixa idade (menos de 13 dias de vida no procedimento). A sobrevida dos pacientes esteve correlacionada à ausência de defeitos associados, estado clínico pré operatório e gradiente residual no local da coarctação da aorta.

A angioplastia, utilizando a artéria carótida como telha para corrigir a coarctação da aorta, associada à hipoplasia do arco aórtico, foi utilizada em 1994 por Mellgreen e Friberg ${ }^{20}$. Nos 13 neonatos tratados com essa técnica, não houve seqüela neurológica em consequiência da ligação da artéria cerebral, nem foram identificados casos de recoarctação no seguimento precoce.

No nosso meio Mendonça e cols. ${ }^{21}$ descreveram nova técnica cirúrgica na qual é possível corrigir a zona de coarctação, sem que haja a perda dos pulsos do membro superior esquerdo.

Um longo e prospectivo estudo multi-institucional envolvendo 326 neonatos, criticamente enfermos, portadores de coarctação da aorta (muitos com defeitos associados) foi realizado por Quaegebeur e cols. ${ }^{22}$, que constataram que a técnica cirúrgica não representou um fator de risco. Também a recoarctação não foi mais freqüente quando se utilizou correção com retalho (patch graft repair). Sobrevida de 24 meses foi obtida em $84 \%$. Também a sobrevida dos pacientes com comunicação interventricular associada teve taxa de sobrevida maior quando se utilizou a correção da coarctação da aorta associada à bandagem da artéria pulmonar, seguida da correção da comunicação interventricular.

Em uma série de 109 pacientes com idade média na cirurgia de 11 anos, Kappetein e cols. ${ }^{23}$ demonstraram que a idade, a bandagem da artéria pulmonar e o tipo de procedimento cirúrgico foram todos fatores preditivos independentes de morte hospitalar. Num seguimento estendido por 35 anos (média de 16,7 anos) foi encontrada taxa de recoarctação relativamente baixa $(5,4 \%)$, entretanto $86 \%$ dos pacientes com anastomose término-terminal apresentaram recoarctação após 30 anos, concluindo que o maior fator preditivo para recoarctação foi a idade, sendo os pacientes com menos de seis meses de vida os que apresentaram a maior taxa de recorrência.

Com o objetivo de determinar a idade ideal para a correção eletiva da coarctação da aorta, Brouwer e cols. ${ }^{24}$ acompanharam 120 pacientes com idade média, na cirurgia, 
de 15 anos, por um período de 44 anos (média de 32 anos). A análise multivariada revelou que a idade na cirurgia foi o único fator de risco para a recoarctação, hipertensão tardia, ou morte prematura. A taxa de recoarctação foi, aproximadamente, de $3 \%$ naqueles pacientes operados aos 15 anos de vida.

Kerdamiel-Ariche e cols. ${ }^{25}$ avaliaram os avanços cirúrgicos e clínicos no resultado do tratamento da coarctação da aorta sem maiores defeitos associados em lactentes com menos de seis meses de vida, concluindo que nos dias atuais a coarctação da aorta deveria ter tratamento cirúrgico precoce, preferentemente pela técnica de Crafoord modificada, no que resultaria em muito poucas complicações hemodinâmicas, sem mortalidade precoce. Já o risco de recoarctação permanece baixo, porém ainda persiste no grupo de neonatos. A médio prazo, o aparecimento de hipertensão ao exercício é um problema freqüente, mesmo naqueles casos com excelentes resultados.

Em experiência de 30 anos envolvendo lactentes e neonatos Zehr e cols. ${ }^{26}$ obtiveram sobrevida, variamdo entre $57 \%$ e $77 \%$ e incidência de recoarctação de $11 \%$ a $27 \%$. Wu e cols. ${ }^{27}$, avaliando os fatores responsáveis pela mortalidade precoce e pela recoarctação em um grupo de 85 neonatos, atribuíram peso, $\mathrm{pH}$, uréia, creatinina e a acidose metabólica, como fatores preditivos da mortalidade precoce, não havendo relação com as dimensões do arco aórtico ou a técnica cirúrgica empregada. Já a recoarctação, ocorrida em $17 \%$, tinha relação com as dimensões do arco transverso.

\section{Tratamento médico: dilatação com cateter balão}

$\mathrm{Na}$ avaliação que se deve fazer para a introdução de nova tecnologia, algumas considerações devem ser levadas em conta: $1^{\circ}$, o procedimento original deve ter grau de mortalidade e morbidade relativamente elevado para permitir que um novo procedimento possa substituí-lo; $2^{\circ}$, o novo procedimento deve apresentar menor fator de risco do que o original e, preferencialmente, deve ser menos invasivo; $3^{\circ}$, o seguimento a curto e longo prazo, de sucessos e também de complicações do novo procedimento deve ser analisado antes de conclusões prematuras, independentes da eficácia e segurança do novo método.

A primeira descrição na literatura do tratamento da coarctação da aorta através da aortoplastia com cateter balão ocorreu em $1982^{28}$. Desde então, centenas de casos têm sido documentados. Praticamente em todos, os resultados com sucesso a curto prazo estão na redução do gradiente e no aumento do diâmetro na região da coarctação da aorta. Também, na maioria dos casos a morbidade relatada e a ocasional perda de pulso parece estar relacionada a cateteres balões de diâmetros aumentados ${ }^{29,30}$. Raramente, existe necessidade de embolectomia após o procedimento ${ }^{30,31}$. A taxa rara de mortalidade durante o procedimento tem sido relatada com a presença de ductus arteriosus associada, ou a perfuração da aorta após o procedimento pela reinserção de cateter angiográfico (pig tail) sem a colocação de guia metálica ${ }^{28}$. Os benefícios a curto prazo, além da abolição do gradiente, são o desaparecimento da hipertensão arterial sistêmica, bem como da hipertensão paradoxal associada ao aumento de catecolaminas ${ }^{32,33}$.

Ainda controversos são os resultados a longo prazo. Enquanto que a maioria de trabalhos descreve a manutenção do aumento do diâmetro na zona de coarctação da aorta $^{34,35}$, outros observam um aumento no gradiente transcoarctação, imediatamente após a dilatação ${ }^{36,37}$. Tambémo resultado parece ser distinto quando a idade do paciente é levada em consideração no procedimento inicial. Alguns trabalhos mencionam que os melhores resultados (redução do gradiente) são obtidos quando a primeira dilatação é realizada após o $1^{\circ}$ ano de vida ${ }^{36}$.

Inicialmente, muitas controvérsias surgiram com relação aos resultados obtidos da dilatação na coarctação da aorta nativa, quando comparados com aqueles da recoarctação. Costaneda-Zuniga e cols. ${ }^{38}$ afirmavam que a recoarctação secundária a uma anastomose término-terminal não apresentavam resultados efetivos à dilatação com cateter balão.

Posteriormente ${ }^{31,39,40}$, verificou-se que é exatamente esse grupo de pacientes que apresenta os melhores resultados a longo prazo. Tynnam e cols. ${ }^{41}$ efetuaram um estudo multicêntrico envolvendo 140 pacientes com idade de três dias a 29 anos. Somente $14 \%$ permaneceram com gradiente $>20 \mathrm{mmHg}$. O diâmetro da coarctação da aorta aumentou de $3,9 \mathrm{~mm}$ para $8,8 \mathrm{~mm}$. Nesse trabalho não foi relatada a ocorrência de aneurismas na zona de coarctação da aorta, sendoassim justificado pela relação diâmetro do balão/ diâmetro da zona de coarctação $<3,0 \mathrm{~mm}$. Em nosso meio, Fontes e cols. ${ }^{42}$ afirmaram que é válida a dilatação da coarctação da aorta nativa com cateter balão. Seu material é composto de 37 pacientes, sendo que 34 com coarctação da aorta nativa. Num seguimento de 13 meses, 21 pacientes encontravam-se assintomáticos. A reavaliação hemodinâmica e angiocardiográfica realizada em 13 pacientes, num período de 12 meses, demonstraram formação aneurismática em um paciente e recoarctação em outro. Concluíram afirmando ser de maior importância a morfologia da coarctação da aorta e o diâmetro do cateter balão para o sucesso do procedimento.

Em 1994, foram publicados dados de 116 pacientes em quatro publicações ${ }^{43-46}$. Em um estudo orientado para comparar a dilatação com cateter balão diretamente com a cirurgia na coarctação da aorta nativa, Huggon e cols. ${ }^{43}$ selecionaram 40 lactentes para a angioplastia e 38 já submetidos à cirurgia. O seguimento foi feito com ecocardiografia, ressonância nuclear magnética e/ou cateterismo cardíaco. A mortalidade precoce e tardia variou de $5 \%$ a $8 \%$ para ambos os grupos. A morbidade do grupo tratado com angioplastia incluiu perda de pulso em $13 \%$ e formação de aneurisma em 7\%. Recoarctação, necessitando reinternação, ocorreu em $30 \%$ dos pacientes submetidos à dilatação com cateter balão e em $18 \%$ daqueles submetidos à cirurgia, sem diferença significativa entre grupos. Os fatores responsáveis pelos maus resultados incluíam a idade e as dimensões do arco aórtico. Em pacientes com menos de 30 dias de vida, a morta- 
lidade foi de $28 \%$ e naqueles com mais de 39 dias de vida de $3 \%$. A taxa de reinternação foi mais alta nos pacientes tratados com cateter balão do que com cirurgia e mais alta ainda em neonatos.

Um segundo trabalho, comparando a dilatação com cateter balão e a cirurgia na coarctação da aorta nativa, foi realizado por Rao e cols. ${ }^{44}$ com um grupo de 29 lactentes com menos de três meses. Quatorze foram submetidos à cirurgia e 15 à aortoplastia com balão. Não houve diferença na mortalidade ( $23 \%$ vs $21 \%$ ) ou alívio imediato do gradiente, bem como na necessidade de reinternação entre os dois grupos. A morbidade incluiu tempo de internação, duração de suporte ventilatório e outras complicações que foram maiores no grupo cirúrgico.

Dados coletados de vários estudos sobre o tratamento cirúrgico ou aortoplastia com cateter balão na coarctação da aorta nativa em pacientes com menos de um ano de vida demonstraram que tanto a mortalidade inicial $(13,5 \%$ para a cirurgia vs $7,0 \%$ para angioplastia) como a mortalidade tardia ( $12,0 \%$ vs $4,2 \%$ respectivamente), foram mais elevadas no grupo cirúrgico. Jáa taxa de recoarctação fora similar $(11,4 \%$ para cirurgia vs $19,0 \%$ para aortoplastia ${ }^{44}$. Entretanto, Johnsone Strauss ${ }^{45}$ acreditam que, possivelmente, os resultados de Rao e cols. ${ }^{44}$ possam ser tendenciosos na seleção dos pacientes para a cirurgia com defeitos associados mais complexos do que aqueles para aortoplastia com cateter balão.

Mendelsohn e cols. ${ }^{46}$ analisaram os resultados da dilatação por cateter balão na coarctação da aorta nativa em um grupo de pacientes maiores (média 6,0 anos, variando entre 0,1 e 19 anos) com seguimento através de estudo hemodinâmico de um a dois anos. Em todos, houve o desaparecimento imediato do gradiente. Durante o seguimento, $64 \%$ apresentaram um gradiente aceitável $(<20 \mathrm{mmHg})$ e $36 \%$ gradientes residuais de mais de $20 \mathrm{mmHg}$, três pacientes desenvolveram aneurisma, ou ambos (um paciente). Recoarctação foi identificada em seis dos 46 pacientes, especialmente naqueles com menos de um ano de vida.

Mais recentemente, Mahestiwari e cols. ${ }^{47}$ analisaram os resultados a longo prazo da angioplastia por cateter balão em lactentes com recoarctação pós-cirúrgica e concluíram ser a angioplastia um procedimento seguro e eficaz a longo prazo. O risco de uma nova recoarctação foi baixo $(16 \%)$ e o tratamento ideal seria uma nova aortoplastia.

A cirurgia ou a aortoplastia por cateter balão, como tratamento de escolha da coarctação da aorta, apresenta a curto prazo resultados comparáveis, embora seu seguimento a longo prazo seja ainda pouco conhecido. Com o objetivo de esclarecer esse problema, Meijboon e cols. ${ }^{48}$ realizaram estudo retrospectivo, avaliando os resultados do tratamento cirúrgico (273 pacientes - idade 1,6 $\pm 2,9$ anos) e da angioplastia por cateter balão (32 pacientes - idade $4,8 \pm 4,6$ anos) na coarctação da aorta em lactentes e crianças ao longo de 23 anos (1977 a 2000). A mortalidade total foi de 7,3\% ocorrendo todos os óbitos no grupo cirúrgico. Em seguimento médio de $11,0 \pm 7,3$ anos, $18 \%$ apresentaram recoarctação no grupo cirúrgico e $12,5 \%$ no grupo da aorto- plastia. Entretanto, na coarctação da aorta nativa, o tratamento cirúrgico apresentou melhores resultados a longo prazo do que a angioplastia.

Em outra publicação esse mesmo grupo ${ }^{49}$ questiona se o manuseio da coarctação da aorta com cateter balão seria diferente na criança e no adulto. Acreditam os autores, bem como grande parte da literatura, que na coarctação da aorta nativa os resultados na criança ou adulto são comparáveis com o uso do cateter balão ou através da cirurgia. Entretanto, os resultados imediatos na coarctação nativa do adulto são melhores com a utilização do cateter balão. Jána recoarctação seria recomendável a aortoplastia por balão no grupo pediátrico.

Os resultados desses e de outros estudos que analisam a aortoplastia com cateter balão na coarctação da aorta não nos parecem ser suficientemente convincentes em demonstrar que a aortoplastia com cateter balão seja um procedimento superior ao tratamento cirúrgico.

Essa, entretanto, não parece ser a experiência de Fontes e cols. ${ }^{50}$, que realizaram a angioplastia com cateter balão em 174 pacientes, sendo 152 portadores de coarctação da aorta nativa. Em função do baixo índice de complicações, pouca invasibilidade, alta hospitalar precoce, custo do procedimento mais baixo que o tratamento cirúrgico, a aortoplastia com cateter balão firma-se como um método de escolha em sua instituição.

\section{Aneurisma pós dilatação}

A dilatação através de cateter balão alivia a coarctação da aorta pelo rompimento da íntima e da média ${ }^{51,52}$. Este é exatamente o mecanismo da abolição da coarctação da aorta, podendo a longo prazo desenvolver a formação de aneurisma. Em duas publicações ${ }^{53,54}$, utilizando-se ressonância nuclear magnética, não foram identificados aneurismas no seguimento tardio dos pacientes. Em um extenso estudo anatomopatológico ${ }^{55}$ de segmentos de coarctação da aorta de pacientes que tinham sido submetidos a angioplastia por cateter balão e posteriormente ressecados durante a cirurgia, foi observado que todos tinham necrose média cística. Isto é indicativo de que a necrose média cística é um achado na coarctação da aorta e que pode representar a base para a formação de aneurisma observado pós angioplastia.

A real incidência de aneurisma pós-tratamento cirúrgico ou através da aortoplastia com cateter balão não está definitivamente estabelecida pela literatura em função de relativamente poucos estudos a longo prazo. Certamente sua incidência não é alta ${ }^{18,41-43,46}$ não devendo ultrapassar 7\% .

A utilização de ultra-som intravascular tem demonstrado ser um método sensível na avaliação das alterações vasculares da aorta, pós aortoplastia com cateter balão. O conhecimento dessas informações morfológicas podem ser úteis na escolha do diâmetro adequado do balão e na identificação e mecanismo de formação de aneurismas pós dilatação. As alterações da íntima identificadas pelo ultra-som intravascular pós dilatação são similares na coarctação da aorta nativa e na recoarctação ${ }^{56}$. O processo de cicatrização e remodelamento de ambos também parecem similar. 


\section{Stents intravasculares}

Os stents intravasculares arteriais ou venosos têm sido usados, já há muitos anos, em adultos e crianças. Entretanto, no tratamento da coarctação da aorta, a utilização de stents intravasculares tem sido muito limitada. Morrow e cols. ${ }^{57}$ realizaram a dilatação de stents intravasculares colocados em 10 suínos 37 dias após a criação cirúrgica de coarctação da aorta. Dois meses pós dilatação e colocação do stent os animais foram submetidos ao cateterismo e angiografia. Os resultados obtidos foram extraordinários, com acentuado aumento da área de coarctação da aorta e normalização do gradiente. Esses resultados demonstraram que nesse modelo animal, a colocação de stent intravascular e a sua dilatação pode ser tratamento coadjuvante da coarctação da aorta. Em situações especiais, a reexpansão dos stents intravasculares pode aumentar as dimensões da aorta, meses após o procedimento inicial.

A experiência com a utilização de stents intravasculares para o tratamento da coarctação da aorta em humanos apresenta resultados a curto prazo bastante promissores ${ }^{58}$. Eles podem representar tratamento alternativo à cirurgia ou angioplastia de portadores de coarctação da aorta (nativa ou recoarctação) ${ }^{59}$.

A utilização de stents em portadores de recoarctação pós-cirurgia parece ser procedimento já estabelecido e aceito pela maioria dos investigadores. A indagação ainda existente seria se na coarctação da aorta nativa este procedimento poderia ser paliativo ou definitivo. Zartner e cols. ${ }^{60}$ utilizaram a colocação de stent em 20 pacientes com idades variando de dois dias a 18 anos e peso entre 2,2 e $63 \mathrm{~kg}$. Desses, cinco apresentavam coarctação da aorta nativa. Não houve complicações nesse grupo de pacientes. Os autores acreditam ser necessárias futuras investigações, particularmente nas lesões nativas, pela possibilidade de se tornar uma terapia definitiva. Um outro estudo, envolvendo número maior de pacientes (64 pacientes) e com seguimento de 7 anos, foi realizado por Segura e cols. ${ }^{61}$. A idade média foi $20 \pm 12$ anos, sendo que 16 haviam sido tratados por cateter balão e cinco por cirurgia. Todos os pacientes tiveram a pressão arterial normalizada, não tendo sido identificadas complicações do tipo aneurisma ou recoarctação nesse período de seguimento. Foi, entretanto, Berger e cols. ${ }^{62}$ que procuraram testar a eficácia da utilização de stents somente na coarctação da aorta nativa, como tratamento inicial. Foram estudados 10 pacientes com idades entre 6,4 a 39,4 anos. A escolha do tamanho do stent foi feita de acordo com o diâmetro da aorta transversa e, também, em função da expectativa do crescimento do paciente. Assim, para os pacientes com idade inferior a 10 anos, o stent escolhido foi de diâmetro de expansão superior a $15 \mathrm{~mm}$. Já nos pacientes com mais de 10 anos, foi utilizadostents com diâmetro de expansão de $25 \mathrm{~mm}$. Após o implante, o gradiente desapareceu totalmente em todos os pacientes. A grande indagação, que permanece, é se com o crescimento do paciente, haverá necessidade de redilatação.
Um dos problemas que vem surgindo no seguimento desses pacientes é o desenvolvimento de reestenose pós colocação do stent, seja na coarctação ou em qualquer outra lesão obstrutiva vascular. Mc Mahon e cols. ${ }^{63}$ avaliaram, retrospectivamente, 368 pacientes com implante de 752 stents. Em 9, a reestenose ocorreu pelo desenvolvimento de proliferação neo-intimal. Acreditam os autores que os fatores de risco envolvidos na reestenose do stent estejam relacionados à técnica de implante, à superposição de stents, à sua angulação e, provavelmente, a uma excessiva distensão do stent.

A colocação de stents intravasculares em crianças, que desenvolvem dissecção aguda pós dilatação, também pode representar uma medida alternativa para o tratamento dessa complicação.

Enfim, o tratamento não cirúrgico da coarctação da aorta está sendo estimulado em muitos centros. A utilização de novos equipamentos de diagnóstico e tratamento, como ultra-som intravascular, cateter balão, bainhas e stent intravascular de menor diâmetro, certamente vão contribuir de maneira extraordinária para o manuseio desses pacientes.

\section{Morbidade}

Já há alguns anos que os investigadores suspeitam que as seqüelas cardiovasculares identificadas no seguimento tardio dos pacientes submetidos ao tratamento cirúrgico da coarctação da aorta não estavam simplesmente relacionadas ao estreitamento anatômico da aorta, mas sim, a uma arteriopatia generalizada. Gardiner e cols. ${ }^{64}$ realizaram interessante estudo da doença vascular em um grupo de 25 pacientes normotensos, submetidos ao tratamento da coarctação da aorta com sucesso, e utilizaram como grupo controle 50 indivíduos normais. Os 25 pacientes tinham sido operados de 7 a 21 anos antes da investigação, que tinha como objetivo determinar de maneira não invasiva se existiam alterações na função da musculatura lisa vascular na artéria braquial direita (pré coarctação) e na artéria femoral (pós coarctação). Em caso afirmativo, se a anormalidade estava relacionada com a idade à cirurgia ou com resposta anormal da pressão ao exercício. Os resultados confirmaram que existem alterações significativas na resposta da resistência dos vasos, a dilatação nos pacientes operados da coarctação da aorta, independente da idade do paciente ou idade à cirurgia. Se esses achados, identificados anos após cirurgia com sucesso da coarctação da aorta, representam uma anomalia congênita ou são secundários a uma hipertensão arterial sistêmica precoce, no momento, não se pode concluir. O que não parece é que a cirurgia ou a aortoplastia precoce não necessariamente impede o desenvolvimento destas anomalias vasculares tardias.

No sentido de avaliar as alterações funcionais pós cirurgia da coarctação da aorta, Johnson e cols. ${ }^{65}$ estudaram o desenvolvimento da massa ventricular esquerda e o desempenho sistólico em dois grupos de crianças. No primeiro, o procedimento cirúrgico foi realizado com idade média de 35 dias, enquanto que no segundo a cirurgia se deu com 
idade média de 8 anos. Utilizando ecocardiografia e ressonância nuclear magnética verificaram que em ambos o índice de massa ventricular esquerda foi maior no primeiro grupo. Um outro achado foi que o desempenho sistólico foi melhor no grupo de lactentes. Sendo assim, a cirurgia precoce pode minimizar ou eliminar anomalias associadas à hipertrofia ventricular esquerda crônica.

Em estudo retrospectivo englobando 114 pacientes, Amaral e cols. ${ }^{66}$ analisaram todos esses parâmetros, relacionando-os à idade na qual a cirurgia foi realizada e concluíram que a reoperação (recoarctação) foi mais freqüentemente identificada, quando realizada em idade mais precoce, ao contrário da hipertensão arterial sistêmica em repouso ou com o exercício, relacionada a cirurgias mais tardias. No seu material também foi infreqüente a limitação física.

\section{Conclusão}

A coarctação da aorta é uma doença que continua a desafiar os investigadores, clínicos e cirurgiões, numa ampla faixa de idade de seus pacientes. Fetos, recém-nascidos e lactentes se apresentam com aspectos particulares no diagnóstico e tratamento, e que muitas vezes podem ser de natureza urgente.

O tratamento com cirurgia, angioplastia com cateter balão, stents intravasculares ou a combinação de terapias podem apresentar obstruções recorrentes ou residuais, que podem, entretanto, ser diagnosticadas e tratadas adequadamente.

Alterações fisiológicas importantes, sob a forma de hipertrofia ventricular esquerda, hipertensão arterial sistêmica ou doença vascular podem surgir muitas décadas após o procedimento inicial.

\section{Referências}

1. Evans W. Congenital stenosis (coarctation), atresia and interruption of the aortic arch: a study of 28 cases. Quarterly J Med 1933; 2: 1-32.

2. Abbott ME. Atlas of Congenital Cardiac Disease. New York: The American Heart Association 1936; 60-61.

3. Sinha SN, Kardatzke ML, Cole RB, et al. Coarctation of the aorta in infancy: pathologic and angiografic measurements. Circulation 1969; 40: 385-98.

4. Edwards JE, Christensen NA, Clagett OT, et al. Pathologic considerations in coarctation of the aorta. Proceedings of Mayo Clinic 1948; 23: 324-32.

5. Ingham DW. Coarctation of the aorta. New York State J Med 1939; 39: 1865-70.

6. Bremer JL. coarctation of the aorta and the aortic isthmus. Arch of Pathol 1948; 45: 425-34.

7. Bonnet LM. Sur la lesion dite stenose congenitale de l'aorte dans la region de l'isthme. Rev Med 1903; 23: 108-225.

8. Bramwell C. Coarctation of the aorta: clinical feature. Br Heart J 1947; 9: 100-24.

9. Reifenstein GH, Levine AS, Grass RE. Coarctation of the aorta: a review of 104 autopsied cases of the "adult type", two years of age or older. Am Heart J 1947; 33: 146-68.

10. Johnson AL, Ferencez C, Wiglesworth FW, et al. Coarctation of the aorta complicated by patency of the ductus (physiologic considerations in the classification of coarctation of the aorta). Circulation 1951; 4: 242-50.

11. Clagett OT, Kirklin JW, Edwards JW. Anatomic variations and pathologic changes in coarctation of the aorta. Surg Gynec and Obstr 1954; 98: 103-14.

12. Rudolph AM, Heimann MA, Spitznas U. hemodynamic considerations in the development of narrowing of the aorta. Am J Cardiol 1972; 30: 514-25.

13. Skoda J. Protokoll der sections - sitzung fur physiologie und pathologie 1855 (cited by Wielenga and Dankneijer, 1968). 19 oct. Wochenblatt der Zeitschrift der Kaiserlich-Koniglichen Gesselshaft der Aerzte zu wien, 710-22.

14. Hutchins GM. Coarctation of the aorta explained as a branch-point of the ductus arteriosus. Am J Path 1971; 63: 203-9.

15. Shinebourne EA, Elseed AM. Relation between fetal flow patterns, coarctation of the aorta and pulmonary blood flow. Br Heart J 1974; 36: 492-8.

16. Crafoord C, Nylin G. Congenital coarctation of the aorta and its surgical treatment. J Thorac Surg 1945; 14: 346-61.

17. Gross RE, Hutnagel CA. Coarctation of the aorta: experimental studies regarding its surgical correction. N Engl J Med 1945; 233: 287-93.

18. Clarkson PM, Nicholson MR, Barrat-Boyes BG, et al. Results after repair of coarctation of the aorta beyond infancy: 10 to 28 years follow-up with particular reference to late systemic hypertension. Am J Cardiol 1983; 51: 1481-8.

19. van Heurn Lwe, Wong CM, Spiegelhalter DJ, et al. Surgical treatment of aortic coarctation in infants younger than three months: 1985-1990. Success of extended end-to-end aortoplasty. J Thorac Cardiovasc Surg 1994; 107: 74-86.

20. Mellgreen B, Friberg LG. Resection of carotid flap: a new surgical technique for newborn children with coarctation of the aorta and critical hypoplasia of the aortic arch. Scand J Thorac Cardiovasc Surg 1994; 28: 49-53.

21. Mendonça JT, Carvalho MR, Costa RK, Filho EF. Coarctation of the aorta: a new surgical technique. J Thorac Cardiovasc Surg 1985; 90: 445-7.

22. Quaegebeur JM, Jonas RA, Weinberg AD, et al. Outcomes in seriously ill neonates with coarctation of the aorta: a multi institutional study. J Thorac Cardiovasc Surg 1994; 108: 841-53.
23. Kappetein AP, Awinderman AH, Bogers AJJC, et al. More than thirty-five years of coarctation repair: an unexpected high relapse rate. J Thorac Cardiovasc Surg 1994; 107: 87-95.

24. Brouwer RMHJ, Erasmus ME, Ebels T, et al. Influence of age on survival, late hypertension and recoarctation in elective aortic coarctation repair. J Thorac Cardiovasc Surg 1994; 108: 525-31.

25. de Kerdaniel-Ariche I, Neville P, Marchand M, et al. Immediate and mid-term results of surgery for aortic coarctation in children under 6 months of age. Arch Mal Coeur Vaiss 1999; 92: 607-12.

26. Zehr KJ, Gillinov AM, Greine PS, et al. Repair of coarctation of the aorta in neonates and infants: a thirty-year experience. Ann Thorac Surg 1995; 59: 33-41.

27. Wu JL, Leung MP, Kareberg J, et al. Surgical repair of coarctation of the aorta in neonates: factors affecting early mortality and recoarctation. Cardiovasc Surg 1995; 3: 573-8.

28. Lock JE, Niemi T, Burke BA, et al. Transcutaneous angioplasty of experimental aortic coarctation. Circulation 1982; 66: 1280-6.

29. Finley JP, Beaulieu RG, Hanton MA, Roy DL. Balloon catheter dilatation of coarctation of the aorta in young infants. Br Heart J 1983; 50: 411-5.

30. Rao PS, Najjar HN, Mardini MK, et al. Balloon angioplasty for coarctation of the aorta: immediate and long-term results. Am Heart J 1988; 115: 657-65.

31. Cooper RS, Ritter SB, Golinko RJ. Balloon dilatation angioplasty: non-surgical management of coarctation of the aorta. Circulation 1984; 70: 903-7.

32. Kan JS, White RI, Mitchell SE, et al. Treatment of restenosis coarctation by percutaneous transluminal angioplasty. Circulation 1983; 68: 1087-94.

33. Choy M, Rocchini AP, Beekman RH, et al. Paradoxical hypertension after repai of coarctation of the aorta in children: balloon angioplasty versus surgical repair. Circulation 1987; 75: 1186-91.

34. Allen HD, Marx GR, Ovitt TW, Goldberg SJ. Balloon dilatation angioplasty for coarctation of the aorta. Am J Cardiol 1986; 57: 828-32.

35. Rao PS. Balloon angioplasty for coarctation of the aorta in infancy. J Pediatr 1987; 110: 713-8.

36. Lock JE, Bass JC, Amplatz K, et al. Balloon dilatation angioplasty of aortic coarctation in infants and children. Circulation 1983; 68: 109-16.

37. Beekman RH, Rocchini AP, Dick MII, et al. Percutaneous balloon angioplasty for native coarctation of the aorta. J Am Coll Cardiol 1987; 10: 1078-84.

38. Castaneda-Zuniga WR, Lock JE, Vlodaver Z, et al. Transluminal dilatation of coarctation of the abdominal aorta. Radiology 1982; 143: 693-7.

39. Hass J, Mooyaart EL, Bush HJ, et al. Percutaneous transluminal balloon angioplasty in restenosis of coarctation of the aorta. Br Heart J 1986; 55: 459-64.

40. Soulen RL, Kan J, Mitchell S, White RI Jr. Evaluation of balloon angioplasty of coarctation restenosis by magnetic resonance imaging. Am J Cardiol 1987; 60: 343-5.

41. Tynan M, Finley JP, Fontes V, et al. Balloon angioplasty for the treatment of native coarctation: results of valvuloplasty and angioplasty of congenital anomalies registry (VACA). Am J Cardiol 1990; 65: 790-2.

42. Fontes VF, Esteves CA, Braga SL, et al. It is valid to dilate native aortic coarctation with a balloon catheter. Int J Cardiol 1990; 3: 311-8.

43. Huggon IC, Quresti AS, Baker EJ, Tynan M. Effect of introducing balloon dilation of native aortic coarctation on overall outcome in infants and children. Am J Cardiol 1994; 73: 799-807. 
44. Rao PS, Chopra PS, Koscik R, et al. Surgical versus balloon therapy for aortic coarctation in infants younger 3 months old. J Am Coll Cardiol 1994; 23: 1479-83.

45. Johnson MC, Strauss AW. The jury is still out regarding balloon therapy for native aortic coarctation. J Am Coll Cardiol 1994; 15: 1589-90.

46. Mendelsohn AM, Lloyd TR, Crowley DC, et al. Late follow-up of balloon angioplasty in children with a native coarctation of the aorta. Am J Cardiol 1994; 74 696-700.

47. Mahestiwari S, Bruckheimer E, Fahey JT, Hellenbraud WE. Balloon angioplasty of post-surgical recoarctation in infants: the risk of restenosis and long-term follow-up. J Am Coll Cardiol 2000; 1: 209-13.

48. Meijboon E, Lekkerkeker RJ, Walhout PA, et al. Comparison of surgical repair with balloon angioplasty for coarctation and recoarctation: a twenty-three year review. Eur Heart J 2001; 22(suppl): 2249.

49. Meijboon E, Plokker THWM, Ernst SMPG, et al. Balloon angioplasty for coarctation of the aorta: should management be different between children and adults? Eur Heart J 2001; 22(suppl): 2252

50. Fontes VF, Esteves CA, Braga SLN, Pedra CAC. Angioplastia com balão no tratamento da coarctação da aorta. Rev Bras Cardiol Invas 2000; 8: 7-15.

51. Sos TA, Sniderman KW, Rettek-Sos B. Percutaneous transluminal dilatation of coarctation of thoracic aorta - post mortem. Lancet 1979; 2: 970-1.

52. Lock JE, Niemit T, Burke BA. Transcutaneous angioplasty of experimental aortic coarctation. Circulation 1982; 66: 1280-6.

53. Boxer RA, La Corte MA, Singh S, et al. Nuclear magnetic resonance imaging in evaluation and follow-up of children treated for coarctation of the aorta. J Am Coll Cardiol 1986; 7: 1095-8

54. Bank ER, Aisen AM, Rocchini AP, Hernandez RJ. Coarctation of the aorta in children undergoing angioplasty: pretreatment and pos-treatment MR imaging. Radiology 1987; 162: 235-40.

55. Isner JM, Donaldson RF, Fulton D, et al. Cystic medial necrosis in coarctation of the aorta: a potential contributing to adverse consequences observed after percutaneous balloon angioplasty of coarctation sites. Circulation 1987; 75: 689-95.

56. Sohn S, Rothman A, Shiota T, et al. Acute and follow-up intravascular ultasound findings after balloon dilatation of coarctation of the aorta. Circulation 1984; 90: 340-7.

57. Morrow WR, Smith VC, Ehler WJ, et al. Balloon angioplasty with stent implantation in experimental coarctation of the aorta. Circulation 1994; 89: 2677-83.

58. Ebeid MR, Prieto LR, Latson LA. Use of balloon expandable stents for coarctation of the aorta: initial results and intermediate-term follow-up. J Am Coll Cardiol 1997; 30: 1847-52

59. Magee AG, Brzezinska-Rajazys G, Qureshi AS, et al. Stent implantation for aortic coarctation and recoarctation. Heart 1999; 82: 600-6.

60. Zartner PA, Schneider BEM, Hausdorft G, et al. Stenting of native or pos operative coarctation. Cardiol Young 2000; 10(suppl 2): 49

61. SeguraJ,Medina A, Viana T, etal.Long-term results of patients with severe coarctation of aorta treated with stent repair: a seven-years study. Circulation 2001; 17: 1088

62. Berger F, Ewert $P$, Beek JVD, et al. Stenting of native coarctation as an alternative to surgery. Cardiol Young 2000, 10(suppl 2): 46.

63. Mc Mahon CJ, Ed-Said HG, Grifka RG, et al. Redilatation of endovascular stents in congenital heart disease: factors implicated in the development of restenosis and neointimal proliferation. J Am Coll Cardiol 2001; 38: 521-6.

64. Gardiner HM, Celermajer DS, Sorensen KE, et al. Arterial reactivity is significantly impaired in normotensive young adults after successful repair of aortic coarctation in childhood. Circulation 1994; 89: 1745-50.

65. Johnson MC, Gutierrez FR, Sekarski DR, et al. Comparison of ventricular mass and function in early versus late repair of coarctation of the aorta. Am J Cardiol 1994; 73: 698-701

66. Amaral F, Granzotti JA, Nunes MA, Somerville J. Quality of life after surgical correction of the aorta coarctation: retrospective analysis of a group of patients with long-term follow-up. Arq Bras Cardiol 1997; 68: 421-7. 